\title{
Research on Scientific Thinking Training Mode of Medical Undergraduate
}

\author{
Zhuo Zhang ${ }^{1, a}$ Meihan Liu ${ }^{1, b}$ Haiyan Zhang $^{1, c *}$ \\ ${ }^{1}$ Urology Department, China Japan Union Hospital of Jilin University, Changchun City, Jilin \\ Province, China, 130033 \\ ${ }^{2}$ Ultrasound Department, China Japan Union Hospital of Jilin University, Changchun City, Jilin \\ Province, China, 130033 \\ ${ }^{3}$ Gastrointestinal Department, China Japan Union Hospital of Jilin University, Changchun City, \\ Jilin Province, China, 130033 \\ Corresponding Author: ZHANG Haiyan,3455064@qq.com
}

Keywords: Scientific Thinking, Training Mode, Medical Undergraduate

\begin{abstract}
In recent years, undergraduate medical education faced with great challenges, with the gradual increase future capacity requirements of medical personnel, five-year duck injection of undergraduate medical education already can not meet the stringent requirements of the new era of community health workers, now stage clinical skills and research only thinking of both clinical medical personnel to come to the fore. Medical undergraduate education students how to develop scientific thinking is a key factor in the future to better integrate into society, so this article will talk about culture experience medical undergraduates' scientific thinking skills, hoping to help students form a correct view of science and values, and improve the quality of their own research.
\end{abstract}

\section{Introduction}

As early as 1969, the Massachusetts Institute of Technology to create. Thereafter, American universities have set up research and undergraduate credits. He won the overwhelming majority of Undergraduate Research credits students think, to participate in scientific research is the most memorable personal experience in undergraduate education, the most important and most valuable experience. In China, undergraduate clinical education objectives clearly stated, by five years in school, should receive an initial capacity of clinical scientific research to meet the future needs of clinical, teaching and research work. Research shows that the culture of thinking, medicine undergraduate, occupies a pivotal position in the undergraduate education. Currently, however, the culture of medical education for undergraduate scientific capacity is a weak link.

\section{The Scientific Thinking Culture Status of Medical Undergraduates}

Clinical Training objectives clearly stated, by learning at the school for five years, should be given an initial clinical scientific ability to adapt to the future needs of clinical, teaching and research work. Visible, research capacity building is one of the professional educational objectives of Education of Medical. At present, the teaching of medical colleges in the country and it is to cultivate scientific ability in teaching graduate and doctoral students in. In undergraduate teaching, scientific ability cultivation is a weak link.

China's current higher medical education, widespread scientific value of medical students lack of knowledge, inadequate attention to scientific research of medical graduates is not high, strong research and awareness of the problem, not written after the vast majority of medical undergraduates medical papers, lack of awareness of scientific research, research and operational capability is not strong, comprehensive analysis ability is poor, this phenomenon is very common medical graduates, which has a great relationship with the current mode of undergraduate teaching in Medical Colleges.

At present, many domestic medical institutions in this regard also made attempts at reform, such 
as undergraduate tutorial system, and actively carry out the second classroom, changing the ratio of school degree credits and research credits and other measures, but with little success. Therefore, in medical colleges and universities based on the actual situation, the establishment of undergraduate research management mechanism, establish a set of performance indicators system Students' Credit and the corresponding reward system, and actively promote scientific and medical undergraduates Research for improving the quality of undergraduate teaching, training and innovative to meet the new requirements of the century, scientific research and medical personnel will have a positive effect.

\section{The Scientific Thinking Training Model of Medical Undergraduates}

Professor of clinical knowledge while introducing progressive approach to scientific problems. Needless to say, first of all medical students need to master clinical skills and knowledge, teaching teachers usually from etiology and clinical manifestations of clinical experience to teach departure system-related diseases, and the patient's condition changes with the analysis and diagnosis of ideas, compare treatment options, such traditional teaching methods of injection and can not achieve the purpose of scientific enlightenment thinking. How limited time to train the theoretical knowledge and scientific thinking combine to effectively inspire students thinking, expand ideas, so as soon as possible to open the door of scientific research, which requires a conscious practice in the teaching process from research point of view to enlighten students' thinking, such as Helicobacter pylori gastritis and gastric cancer risk factors, in addition to master the clinical significance of this indicator, the Tutors may also explain Hp disease pathology students from the perspective of the main mechanisms leading to gastric mucosal barrier disruption and pathogenic target molecule, then extended to Hp vaccine and subsequent development prospects at home and abroad. This will not only enrich the students' knowledge and to be progressive approach to enable students to understand information about current research frontier. At the same time for certain diseases, the content of textbooks is relatively old, teaching teachers can consciously on the latest research trends of the disease, the latest publication from the genes related to the international community may be involved in the occurrence and development of important therapeutic targets in the form of science professors to students, enabling them to focus on the direction of research and research models have a preliminary understanding.

Turn diversified teaching mode, develop research and innovation thinking. Education is the only method of clinical teaching process tasted the effect is not ideal, it is recommended to open a wide range of teaching, learning clinical teaching is the most basic, of course, in addition to research and recommend organizational interest groups to participate in master of the discipline thesis report, literature review reports, papers report writing methods and techniques, graduate thesis reports. At the same time set up a week or two and discuss scientific issues, give reviews after training students to find their own literature, summarized the ability to encourage students to speak freely, and teachers in teaching students to clarify their own understanding of the issues and ideas, guide students involved in early research and creative thinking training to enhance students' awareness of innovation and practical ability. Established scientific issues may be directed against a particular disease may be for some cutting-edge academic point of view, often seemingly a common problem, it is likely to become the prototype of a scientific proposition. In addition, students participate in research practice is extremely important way to foster creative thinking and ability, students can also If you are interested to learn some basic laboratory experimental techniques, to enable students to further scientific research has perceptual awareness, encourage undergraduate final students go to school more organized various research seminars, involving a wide range of research areas, can communicate through seminars and with experts in related fields, in order to find the direction of interest and research ideas. And to take a wide range of assessment modes, not just stick to master knowledge or examination of the books, should be combined with the students' capability of independent innovation and learning initiative is given a comprehensive evaluation of students usually summarize test results, practical skills and research initiative develop scientific evaluation mechanism, which is not only beneficial to explore students 'interest in scientific research, but also 
can enhance students' mastery, analyze problems, problem-solving skills, and foster the development of research and innovation thinking ability.

Focus on training students' ability to teamwork and academic exchanges. In the process of clinical teaching, I found many of the comprehensive ability of students often lack the trust of teamwork and academic exchanges enthusiasm, so behind closed doors, is not conducive to training mode complacent expand scientific thinking. First, whether it is scientific or clinical diagnosis and treatment process teamwork are essential, but the strength of individual ability to obtain one of the decisive factors for success in the organization, group discussions, access to information and summarize the process learn from each other but also help improve the overall quality of students, students to think independently while strengthening collaboration with the training organization and management capacity in order to meet the development requirements of the times to become high-quality personnel. In addition, foreign academic exchange capacity is also clinical teaching process needs to focus on training, teaching teachers according to the actual situation, to carry out some small forums or academic salons, in an atmosphere of good research students while providing direct communication opportunity to encourage students to report academic research results in the form of the show, and even invite some experts in the field of guidance, and to give professional advice and recommendations, as far as possible within a limited period of time so that students benefit. At the same time consciously train students' ability to write papers, the paper form without formality, as long as can clearly explain their academic views and scientific thinking are encouraged. For some of the better papers, teaching teachers can further professional guidance, help students to express, so more conducive to mobilize the students' research initiative.

Research long and arduous road, want to be successful in the area of scientific research, it must have a hard-working, not afraid of failure. The history of those amazing achievements in scientific research is not done overnight, but often after long-term accumulation of research and a lot of people together to study hard in order to gain something, there are many scholars even in their lifetime can not be sure, and in the years to witness history after only recognized by people, and this insistence are derived from their ideal stick for the love of work and right. More important is to establish realistic scientific spirit, adhere to rigorous scientific approach and the correct orientation and research excellence scholarship style and these are becoming qualified researchers. All fork failure and frustration are the only way to success, therefore, whether in work or in medical science students must persevere in innovation, the spirit of seeking truth from facts that will make them lifelong benefit.

\section{The Specific Measures of Scientific Thinking Training for Medical Undergraduates}

The Creation of Research-Related Courses. In the normal teaching, the creation of basic research elective courses, such as "Medical Document Retrieval and Essay Writing", "Preventive Medicine", "Statistics", so that students master the literature review, research topics, experimental design, data collection, thesis writing and other basic skills. At the same time, the creation of "microbiology experiment science", "molecular biology" and other experimental techniques courses, students commonly used in medical research methods and techniques to understand.

Held "Doctor Forum" and the Scientific Academic Lectures. In order to better develop undergraduate academic horizons and take advantage of Teachers College, the College organizes regular "Doctor Forum" and invited to study the return of Dr. and Dr. graduated alumni, undergraduate academic lectures. To introduce undergraduate forefront of medical knowledge, broaden their academic horizons. In addition, we also held a number of research and research design ideas lectures regularly to the research team as a unit to carry out academic exchange activities to encourage students to listen, to enable students to gradually master the basic research program, research paper writing conventions familiar to stimulate students' interest in scientific research activities.

Organizational Experiments and Research. Under the guidance of teachers and strict requirements, master experimental operation, familiar with the basic experimental skills. Students use their holidays, summer vacation and other time, experimental operation of the project feasibility 
report and its protocols. Students due to the relatively unfamiliar scientific research, produce a variety of questions, conduct experiments irregular operations, thus affecting the results. During the experiment, student experiment at any time to report progress, teachers pay close attention to the progress of the experiment the students to complete predetermined research objectives. In addition, also allows students to "assistant" role, participation of teachers' research projects.

Paper Writing and Guidance. Students According to the results, refer to the latest progress of the research, in the form of research papers writing papers. After the completion of the first draft of the paper, research paper writing can be the first by a relatively familiar with the high school students to modify, and then the teacher changes. Participation by modifying the thesis, the student's paper writing skills improved. At last, participate in the "Challenge Cup" College Students' extracurricular academic science and technology work competition, or contributor to professional journals, or compiled into a school outstanding research report, to encourage students to research enthusiasm.

\section{Conclusion}

Medical undergraduate education is not limited to training in clinical skills, but should aim to foster innovation and scientific thinking skills of both clinical medical personnel, they will not only be able to better approaches to science into clinical practice to urgent problems, the more acutely aware of the clinic. Thus, casting a large number of elite medical back-up, to cultivate their scientific thinking, is imperative.

\section{References}

[1] Jia Xinzhang, Li Jingyuan. Preclinical Medical Education, Vol. 6 (2014) No 53, p.25-26

[2] Peng Sue, Wang Qunyong. Guangdong Medical College, Vol. 12 (2015) No 27, p.74-76

[3] Qian Xiyuan, Jing Jianfen, Hou XuSiem. Medical Education, Vol. 30 (2014) No 19, p.144-145

[4] Wang Kuailiang. Preclinical Medical Education, Vol. 29 (2008) No 27, p.21-23

[5] Zhang Gongxu, Sun Jing. Medical Education, Vol. 8 (2013) No 27, p.57-60 